\title{
Development of telmisartan in the therapy of spinal cord injury: pre-clinical study in rats
}

This article was published in the following Dove Press journal:

Drug Design, Development and Therapy

14 August 2015

Number of times this article has been viewed

\section{Chien-Min Lin ${ }^{1, *}$ \\ Jo-Ting Tsai ${ }^{2, *}$ \\ Chen Kuei Chang' \\ Juei-Tang Cheng ${ }^{3}$ \\ Jia-Wei Lin'}

'Department of Neurosurgery, ${ }^{2}$ Department of Radiation Oncology, Shuang Ho Hospital-Taipei Medical University, ${ }^{3}$ Institute of Medical Science, College of Health Science, Chang Jung Christian University,

Tainan City, Taiwan

*These authors contributed equally to this work
Correspondence: Jia-Wei Lin Department of Neurosurgery, Shuang Ho Hospital-Taipei Medical University, Taipei City I I03I, Taiwan

Tel +886222490088 ext 8866

Fax +886222480900

Email ns246@tmu.edu.tw
Background: Decrease of peroxisome proliferator-activated receptors- $\delta$ (PPAR $\delta$ ) expression has been observed after spinal cord injury (SCI). Increase of PPAR $\delta$ may improve the damage in SCI. Telmisartan, the antihypertensive agent, has been mentioned to increase the expression of PPAR $\delta$. Thus, we are going to screen the effectiveness of telmisartan in SCI for the development of it in clinical application.

Methods: In the present study, we used compressive SCI in rats. Telmisartan was then used to evaluate the influence in rats after SCI. Change in PPAR $\delta$ expression was identified by Western blots. Also, behavioral tests were performed to check the recovery of damage.

Results: Recovery of damage from SCI was observed in telmisartan-treated rats. Additionally, this action of telmisartan was inhibited by GSK0660 at the dose sufficient to block PPAR $\delta$. However, metformin at the dose enough to activate adenosine monophosphate-activated protein kinase failed to produce similar action as telmisartan. Thus, mediation of adenosine monophosphate-activated protein kinase in this action of telmisartan can be rule out. Moreover, telmisartan reversed the expressions of PPAR $\delta$ in rats with SCI.

Conclusion: The obtained data suggest that telmisartan can improve the damage of SCI in rats through an increase in PPAR $\delta$ expression. Thus, telmisartan is useful to be developed as an agent in the therapy of SCI.

Keywords: PPAR $\delta$, AMPK, spinal cord injury, angiotensin receptor blocker, metformin

\section{Introduction}

Spinal cord injury (SCI) is defined as a damage to the spinal structure and/or function that can be caused by a host of etiological factors, including labor injuries and traffic accidents; the condition also creates enormous physical and emotional cost to individuals. ${ }^{1}$ SCI easily leads to motor paralysis and sensory dysfunction while both afferent sensory and efferent motor innervations are passing through the spinal cord. ${ }^{2}$ The sensory dysfunction is associated with urinary impairment, which is a major factor in morbidity and even mortality in those with SCI. ${ }^{3}$

Peroxisome proliferator-activated receptors (PPARs) are ligand-activated transcription factors belonging to the nuclear hormone receptor superfamily, which includes the classical steroid, thyroid, and retinoid hormone receptors. ${ }^{4}$ At present, three PPAR subtypes have been identified to be designed as PPAR $\alpha, \operatorname{PPAR} \delta$, and PPAR $\gamma .{ }^{5}$ PPARs are involved in the pathogenesis of several diseases, including diabetes mellitus, obesity, atherosclerosis, neurological diseases, and SCI. ${ }^{6-8}$ It has been documented that GW0742 (a selective agonist of PPAR $\delta$ ) can reduce the development of inflammation and tissue injury associated with SCI. ${ }^{9}$ However, application of useful medication in patients with SCI is still not enough.

Angiotensin II (Ang II) receptor blockers (ARBs) are commonly used to lower the blood pressure. ${ }^{10}$ In addition to antihypertensive effect, several clinical trials show 
that ARBs have some effects on energy metabolism. ${ }^{11}$ The pleiotropic effects of ARBs include the upregulation of uncoupling protein 1 or Ang II receptor type 1 expression and the activation of PPAR. ${ }^{12}$ Recently, telmisartan, one of the ARBs, has been found to increase the expressions of PPAR $\delta$ and adenosine monophosphate-activated protein kinase (AMPK) in skeletal muscle of rats. ${ }^{13}$ Additionally, the activation of PPAR $\delta$ is helpful to improve the injury from SCI in diabetic rats. ${ }^{14}$ The present study is thus designed to investigate the therapeutic potential of telmisartan in SCI using animal model as pre-clinical evidence.

\section{Materials and methods Experimental animals}

The male Wistar rats obtained from the animal center of National Cheng Kung University Medical College (Tainan, Taiwan) were maintained in a temperature-controlled room $\left(25^{\circ} \mathrm{C} \pm 1^{\circ} \mathrm{C}\right)$ under a 12 -hour light-dark cycle (lights on at $06 \mathrm{am}$ ). All rats received water and standard chow (Purina Mills, LLC, St Louis, MO, USA) ad libitum. All animalhandling procedures were performed according to the Guide for the Care and Use of Laboratory Animals of the National Institutes of Health and followed the guidelines of the Animal Welfare Act.

\section{Spinal cord injury}

We performed the SCI mainly according to our previous method ${ }^{15}$ with some modifications. ${ }^{16}$ In brief, the laminectomy for removal of the vertebral peduncle was performed between T8 and T9 on rats under anesthesia with sodium pentobarbital (30 mg/kg, intraperitoneally; Sigma Chemical Co, St Louis, MO, USA). We used a calibrated aneurysm clip with a closing pressure of $55 \mathrm{~g}$ to place between the dorsal and ventral surfaces of spinal cord for 1 minute. Animals that received the same laminectomy without compression with clip were grouped as the sham-operated control. Then, we injected $0.1 \mathrm{~mL}$ of cefazolin $(10 \mathrm{mg} / \mathrm{kg}$ body weight; China Chemical Pharmaceutical Co, Ltd, Taipei, Taiwan) into all animals for 3 days after the surgery. Animals that received SCI were individually housed on a special bedding to prevent the formation of pressure sores. Additionally, rats had their bowel and bladder manually compressed twice daily. Food and water were supplied at a lowered height in their cages for free accessibility. ${ }^{15}$ Similar to the previous report, ${ }^{15}$ the rats typically did not survive beyond 4-5 weeks after the SCI. In the present study, telmisartan, metformin and GSK0660 (a selective antagonist of PPAR $\delta$ ) purchased from Tocris Bioscience (Ellisville, MO, USA) were dissolved in dimethyl sulfoxide and diluted with saline. The oral solution of metformin $(320 \mathrm{mg} / \mathrm{kg}),{ }^{17}$ telmisartan $(5 \mathrm{mg} / \mathrm{kg}),{ }^{13,18}$ and $/$ or GSK0660 $(0.1 \mathrm{mg} / \mathrm{kg})^{14}$ was intravenously injected into the rats via tail vein for 20 days after 1 week of SCI every morning (10 am). Additionally, we employed the vehicle at the same volume to treat the control group in the same manner. The behavioral test took place every Monday evening (6 pm) after 1 week of drug administration. The rats that cannot survive until the end of this study (28 days) were excluded. The results were obtained from each group of six rats for further analysis.

\section{Locomotor scale}

According to the earlier report, ${ }^{19}$ the Basso, Beattie and Bresnahan (BBB) locomotor rating scale (locomotor scale) from 0 to 21, where 0 reflects no locomotor function and 21 reflects normal performance, is used to evaluate the effects of metformin $(320 \mathrm{mg} / \mathrm{kg}),{ }^{17}$ telmisartan $(5 \mathrm{mg} / \mathrm{kg}),{ }^{13}$ and $/$ or GSK0660 $(0.1 \mathrm{mg} / \mathrm{kg})^{14}$ on functional recovery after SCI. We arranged the rats to walk around freely in a $90 \mathrm{~cm}^{2}$ field (width and length) for 4 minutes, and movements of the hind limb were observed continuously. Rats were trained to gently adapt to the field at first. Two investigators conducted 4-minute testing sessions on each leg of the rats that walked continuously in the field. This study started one day postinjury and continued for 20 days or over. The functional deficits were double-blind checked by the trained investigators. The results of behavior outcomes and examples of locomotor scores were also recorded in the digital video.

\section{Inclined plane test}

We applied the inclined plane test (IPT) to evaluate the ability of rats to maintain their position for 5 seconds on an inclined plane that was covered with a rubber mat containing horizontal ridges ( $1 \mathrm{~mm}$ deep, spaced $3 \mathrm{~mm}$ apart, and selfmade), as described previously. ${ }^{20}$ The rats were determined as the angle of the surface was increased from $5^{\circ}$ to $90^{\circ}$ at $5^{\circ}$ intervals. The angle at which the rat could not maintain its position was the outcome measured.

\section{Limb hanging test}

This test is used for evaluating both forelimb and hind limb function. However, it is mainly employed for testing muscle function in the forelimbs of animals with SCI, as mentioned in previous reports. ${ }^{21}$ The test is conducted using a $12 \mathrm{~cm}$ long and $1.8 \mathrm{~mm}$ wide rounded metal rod that is applied to the volar surface of the forepaw, and the presence or absence of grasping and the release time in seconds are recorded. When the rod is lifted above the surface and suspended, the testing of animal's forelimb muscle strength is possible. In addition, contact of the body, hind limb, or tail with the ground or 
parts of the equipment on the sides should be prevented. The time that the rat hangs suspended on the rod is measured and recorded. This testing should typically be repeated five times. The mean values of each administration were calculated, as described previously. ${ }^{21}$

\section{Pain test}

All rats were adapted to testing chambers for at least 2 days. The occurrence of foot withdrawal in response to the mechanical notching of the plantar surface of each hind paw was measured using a flat-tipped cylindrical probe that measured $200 \mu \mathrm{m}$ in diameter, following previous methods. ${ }^{22}$ Von Frey filaments capable of exerting forces with a uniform tip diameter were applied to a designated loci on the plantar surface of the foot. During each experiment, the rat was placed in an obvious plastic cage with a floor of wire with approximately $1 \mathrm{~cm}^{2}$ openings. The cage was elevated so that stimulation was practical for each hind foot from underneath the rodent. The duration of each stimulus was approximately 1 second, and the interstimulus interval was approximately $10-15$ seconds. The force unit was expressed in Newtons $(\mathrm{N})$.

\section{Western blotting analysis}

Spinal cord tissues were isolated from all the six rats in each group treated with telmisartan and/or GSK0660, and metformin after SCI. Tissues were homogenized in the ice-cold buffer solution containing $10 \mathrm{mM}$ Tris- $\mathrm{HCl}(\mathrm{pH} 7.4), 20 \mathrm{mM}$ ethylenediaminetetraacetic acid, $10 \mathrm{mM}$ ethylene glycol tetraacetic acid, $20 \mathrm{mM} \beta$-glycerophosphate, $50 \mathrm{mM} \mathrm{NaF}$, $50 \mathrm{mM}$ sodium pyrophosphate, $1 \mathrm{mM}$ phenylmethylsulfonyl fluoride, and the protease inhibitors $25 \mu \mathrm{g} / \mathrm{mL}$ leupeptin and $25 \mu \mathrm{g} / \mathrm{mL}$ aprotinin. The mixture was then centrifuged at $1,000 \times g$ for 10 minutes. The obtained supernatant was further centrifuged at $48,000 \times g$ for 30 minutes. After resuspension of the pellet in ice-cold Triton X-100 lysis buffer, samples were centrifuged at $14,010 \times g$ for 20 minutes. All the above centrifugations were performed at $4{ }^{\circ} \mathrm{C}$. The supernatant was collected in an Eppendorf tube to store at $-80^{\circ} \mathrm{C}$. The membrane extracts $(20-80 \mu \mathrm{g})$ in the supernatant were applied for separation using $10 \%$ sodium dodecyl sulfatepolyacrylamide gel electrophoresis. The obtained proteins were transferred onto a BioTrace ${ }^{\mathrm{TM}}$ polyvinylidene fluoride membrane (Pall Corporation, Pensacola, FL, USA) for 2 hours. The blots were developed through the reaction with primary antibodies $(1: 1,000)$ of receptor for advanced glycation endproducts (RAGE), high-mobility group box 1 protein (HMGB1), phosphorylated AMPK (p-AMPK), PPAR $\delta$, and actin (Abcam, Cambridge, UK) for 16 hours. Then, they were hybridized with horseradish peroxidase-conjugated rabbit anti-rabbit IgG (Jackson ImmunoResearch Laboratories, Inc, PA, USA) for 2 hours and developed with the Western Lightning Chemiluminescence Reagent PLUS (PerkinElmer Life Sciences Inc., Boston, MA, USA). We employed Gel-Pro analyzer software 4.0 (Media Cybernetics, Silver Spring, MD, USA) to quantify the densities of the obtained immunoblots at $35 \mathrm{KDa}$ for RAGE, $29 \mathrm{KDa}$ for HMGB1, $62 \mathrm{KDa}$ for p-AMPK, $40 \mathrm{KDa}$ for PPAR $\delta$, and $43 \mathrm{KDa}$ for actin, respectively.

\section{Statistical analysis}

All results were expressed as the mean \pm standard error of each group. Statistical analysis was performed using analysis of variance with the Newman-Keuls post-hoc. A $P$-value of $\leq 0.05$ was considered statistically significant.

\section{Results Effects of telmisartan on motor function and pain response in rats with $\mathrm{SCl}$}

Overground locomotion using the BBB scoring system showed consistent weight support and consistent forelimbhindlimb coordination. ${ }^{23}$ As shown in Figure 1A, telmisartan improved the BBB locomotor scale in rats with SCI.

The inclined plane evaluates the ability of the animal to maintain its body position on a surface that is gradually raised to increasing angles. In the rat models of SCI, this test has been shown reliable, consistent, and sensitive and that has been used to assess the therapeutic modalities. ${ }^{24}$ As shown in Figure 1B, telmisartan improved the behaviors including the result of IPT in rats with SCI.

Limb hanging wire test evidenced a reduction of muscle strength in rats. ${ }^{25}$ As shown in Figure 1C, telmisartan improved the results of limb hanging test in rats with SCI.

Pain test evaluates the nociceptive mechanical threshold. As shown in Figure 1D, telmisartan decreased the mechanical threshold in rats with SCI.

As shown in Figure 1, telmisartan combined injection of GSK0660 (0.1 mg/kg, once daily) to these rats that attenuated the improvements of motor function and pain responses induced by telmisartan.

\section{Effects of telmisartan on PPAR $\delta$ and $\mathrm{P}$-AMPK expressions in rats with $\mathrm{SCl}$}

After evaluating the behavioral tests, we used the spinal cord from each rat in the same group to perform the Western blotting analysis. As shown in Figure 2, the PPAR $\delta$ and p-AMPK expressions in spinal cord of SCI rats were markedly lower than that in normal rats. The expressions of PPAR $\delta$ and p-AMPK were both reversed by telmisartan in 
A

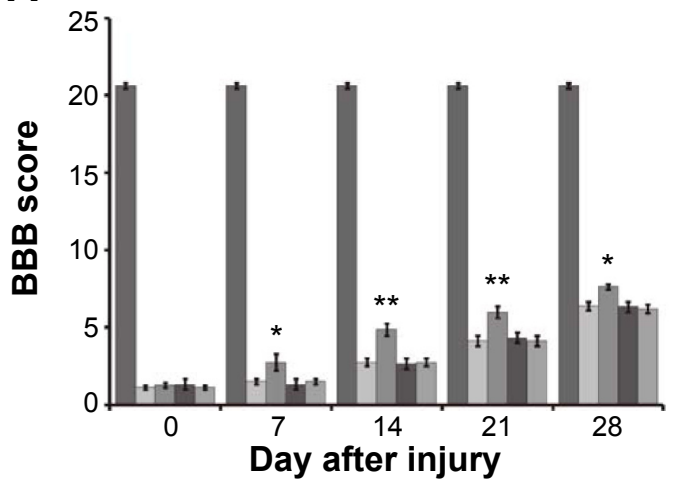

C

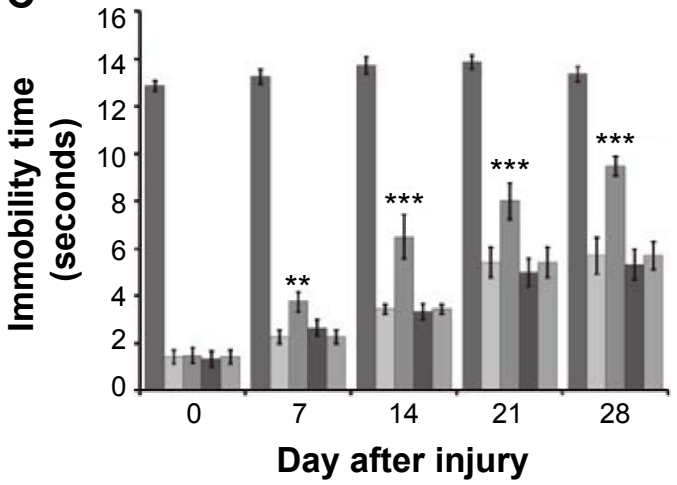

B

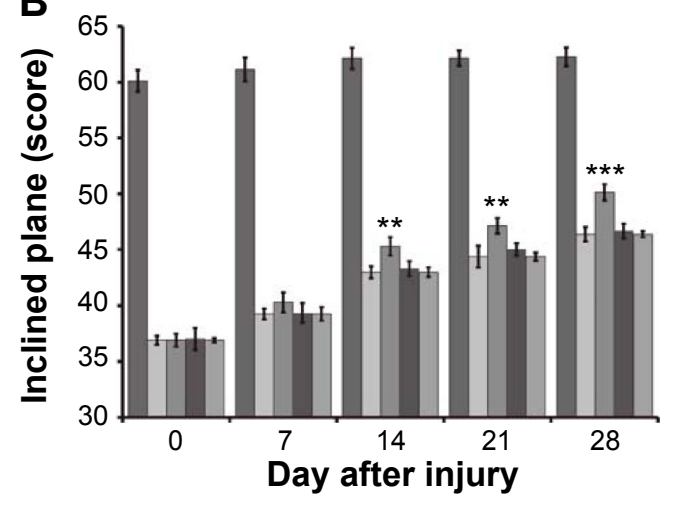

D

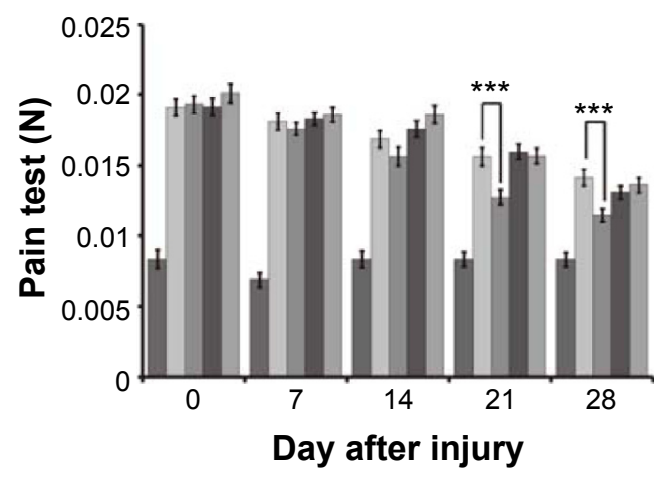

- Control $\square \mathrm{SCl} \backsim \mathrm{SCl}+$ telmisartan $\because \mathrm{SCl}+\mathrm{GSK}+$ telmisartan $\because \mathrm{SCl}+\mathrm{GSK}$

Figure I Changes in behavioral and pain tests in rats with $\mathrm{SCl}$ after receiving telmisartan and/or GSK0660.

Notes: The rats with SCl were treated with telmisartan $(5 \mathrm{mg} / \mathrm{kg})$ and/or GSK0660 $(0.1 \mathrm{mg} / \mathrm{kg})$ intravenously once daily for 28 days. Tests showed (A) BBB locomotor scale, (B) inclined plane test, $(\mathbf{C})$ limb hanging test, and (D) pain test. Values (mean $\pm \mathrm{SE}$ ) were obtained from each group of six rats. $* P<0.05$, $* * P<0.0 \mathrm{I}$ and $* * * P<0.00 \mathrm{I}$ compared with the vehicle-treated $\mathrm{SCl}$ rats.

Abbreviations: BBB, Basso, Beattie and Bresnahan; GSK, glycogen synthase kinase; SCl, spinal cord injury; SE, standard error.

A
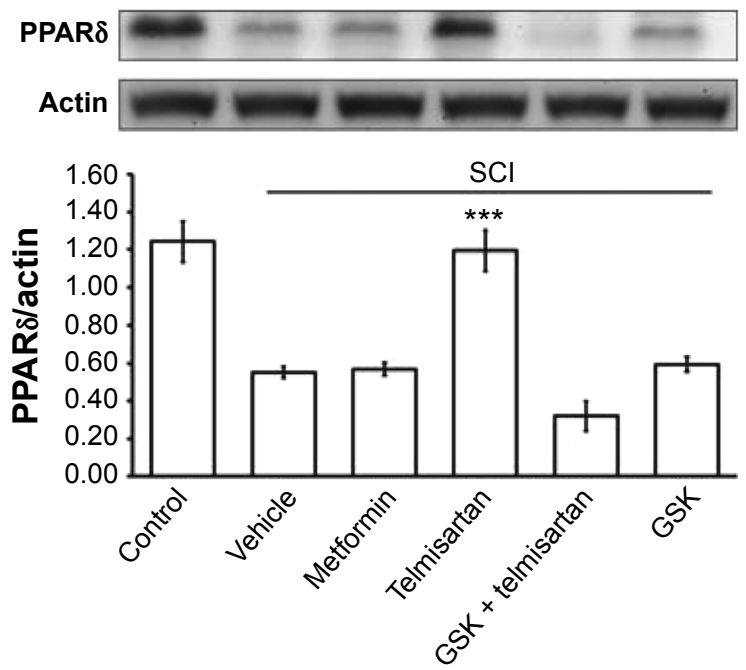

B
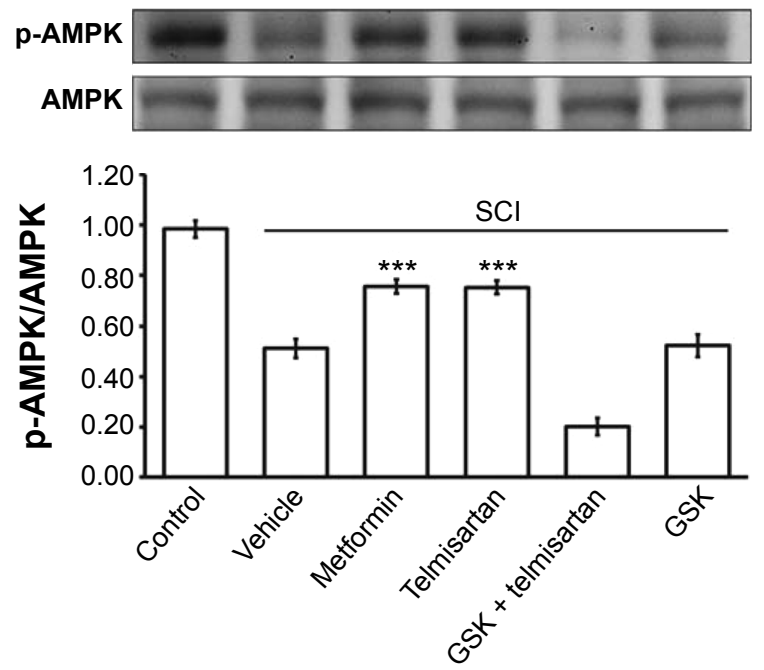

Figure 2 The expressions of PPAR $\delta$ and P-AMPK in spinal cord of rats with SCl after treatment with metformin, telmisartan and/or GSK0660.

Notes: The rats with SCl were treated with metformin $(320 \mathrm{mg} / \mathrm{kg})$, telmisartan $(5 \mathrm{mg} / \mathrm{kg})$ and/or GSK0660 $(0.1 \mathrm{mg} / \mathrm{kg})$ intravenously once daily for $28 \mathrm{days}$. Expressions of PPAR $(\mathbf{A})$ and P-AMPK (B) were identified using Western blotting analysis. Protein expression was normalized against that of $\beta$-actin used as a loading control. Data represent the mean \pm SEM of six animals. $* * * P<0.001$ compared with the vehicle-treated $\mathrm{SCl}$ rats.

Abbreviations: AMPK, adenosine monophosphate-activated protein kinase; GSK, glycogen synthase kinase; p-AMPK, phosphorylated AMPK; PPAR, Peroxisome proliferatoractivated receptors; SCl, spinal cord injury; SEM, standard error of mean. 
rats with SCI. This action of telmisartan was attenuated by GSK0660 at an effective dose to block PPAR $\delta$ as described previously. ${ }^{13}$ However, metformin failed to modify the expression of PPAR $\delta$ in rats with SCI.

\section{Metformin failed to improve the motor function and pain response in rats with $\mathrm{SCl}$}

As shown in Figure 3, metformin failed to improve the behaviors in rats with SCI including the BBB locomotor scale, IPT, limb hanging test and pain test. The treated dose of metformin is enough to increase p-AMPK in these rats as shown in Figure 2B.

\section{Effects of telmisartan on HMGBI and RAGE expressions in rats with $\mathrm{SCl}$}

PPAR $\delta$ has been indicated to show anti-inflammatory effects in neurological and inflammatory-related diseases. ${ }^{26}$ As shown in Figure 4, the inflammatory indicators, HMGB1 and RAGE expressions, in the spinal cords of rats with SCI were
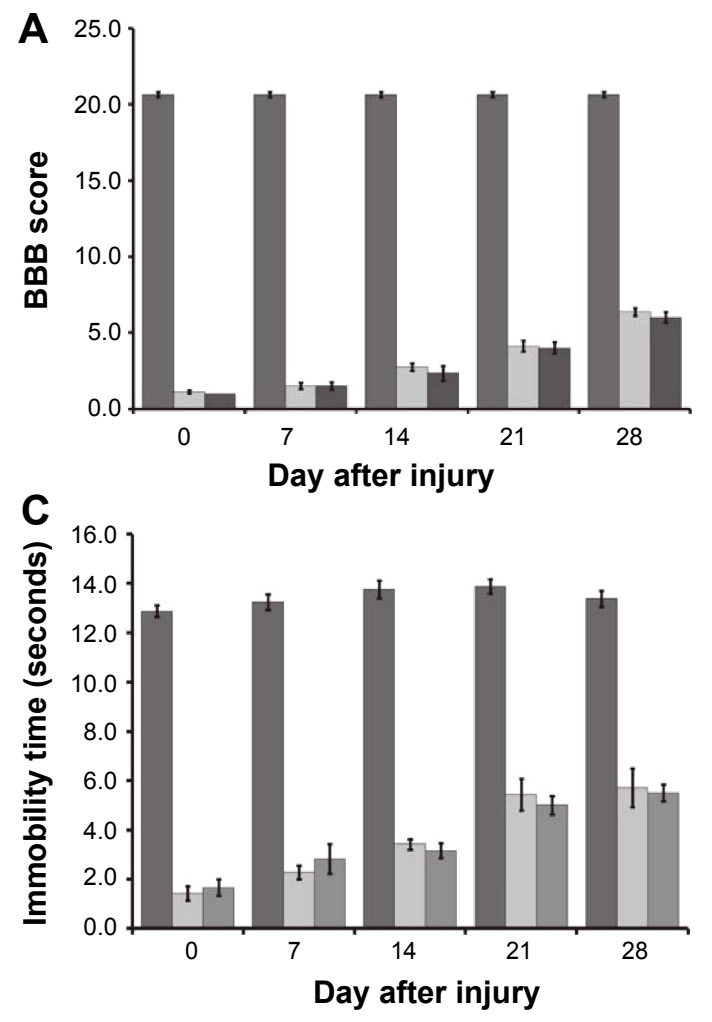

markedly higher than that in normal rats. The expression of HMGB1 (Figure 4A) or RAGE (Figure 4B) was reversed by telmisartan in rats with SCI. This action of telmisartan was also attenuated by GSK0660. Additionally, as shown in Figure 4, metformin failed to modify the expressions of HMGB1 and RAGE in rats with SCI.

\section{Discussion}

In the present study, we found that the motor dysfunction and pain responses were improved by telmisartan in rats with SCI. This action of telmisartan was inhibited by a blockade of PPAR $\delta$. PPAR $\delta$ expression was not reduced after SCI in rats treated with telmisartan. Moreover, the expression of p-AMPK was also raised by telmisartan. Metformin at the dose enough to activate AMPK failed to modify the motor dysfunction and pain responses in rats with SCI. The therapeutic potential of telmisartan in rats with SCI is mainly through the restoration of PPAR $\delta$ expression.

Recently, the pharmacological activation of PPAR $\delta$ has been considered as the potential target due to its
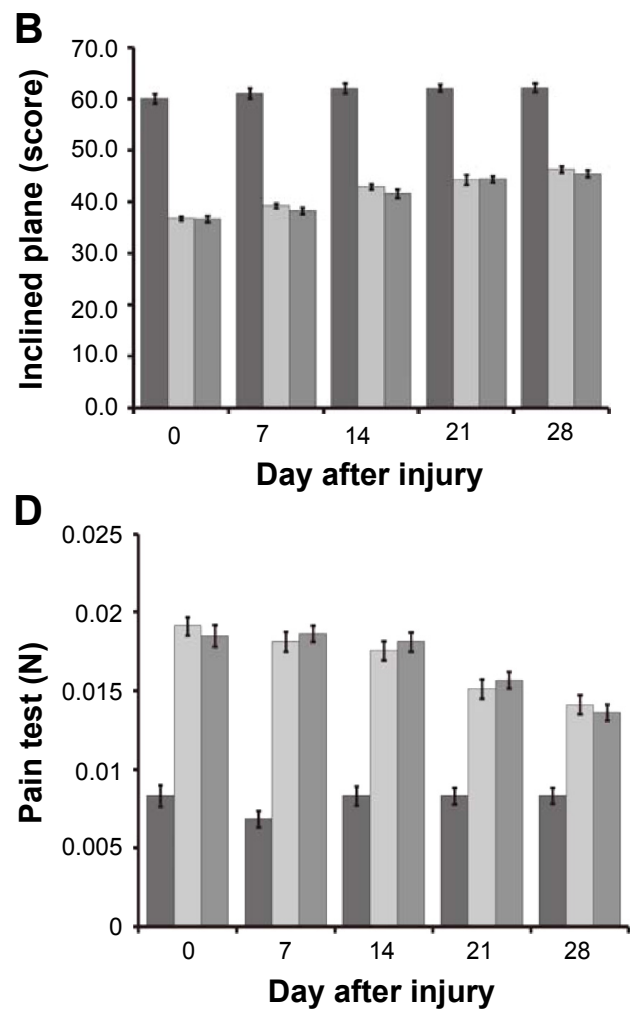

\section{- Control $\quad \mathrm{SCl} \quad \mathrm{SCl}+$ melformin}

Figure 3 Changes of behavioral and pain tests in rats with $\mathrm{SCl}$ by metformin.

Notes: The rats receiving SCl was further treated with metformin $(320 \mathrm{mg} / \mathrm{kg})$ intravenously once daily for 28 days. Responses show (A) BBB locomotor scale, (B) inclined plane test, (C) limb hanging test, and (D) pain test. Values (mean \pm SE) were obtained from each group of six rats. Each group compared with the vehicle-treated SCI rats without statistical difference.

Abbreviations: $\mathrm{BBB}$, Basso, Beattie and Bresnahan; $\mathrm{SCl}$, spinal cord injury; SE, standard error. 
A

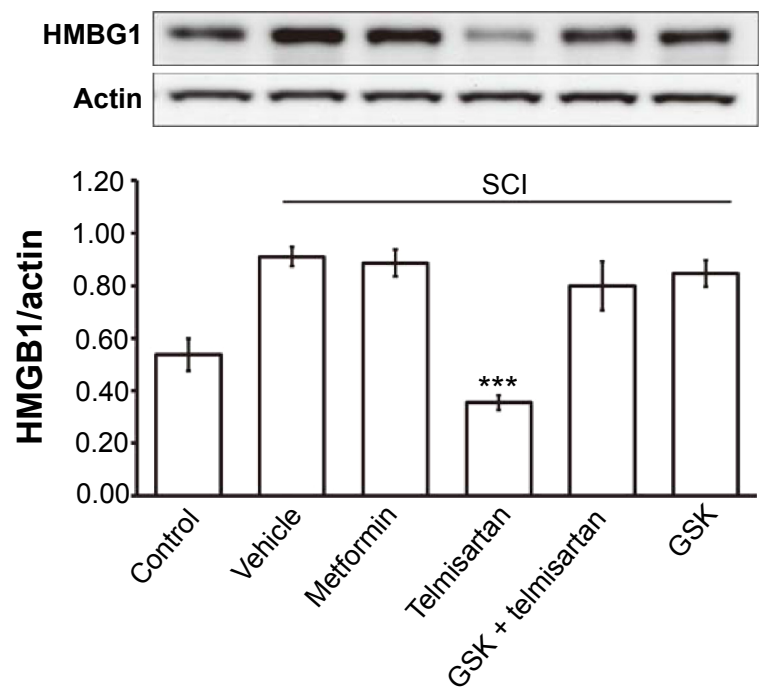

B

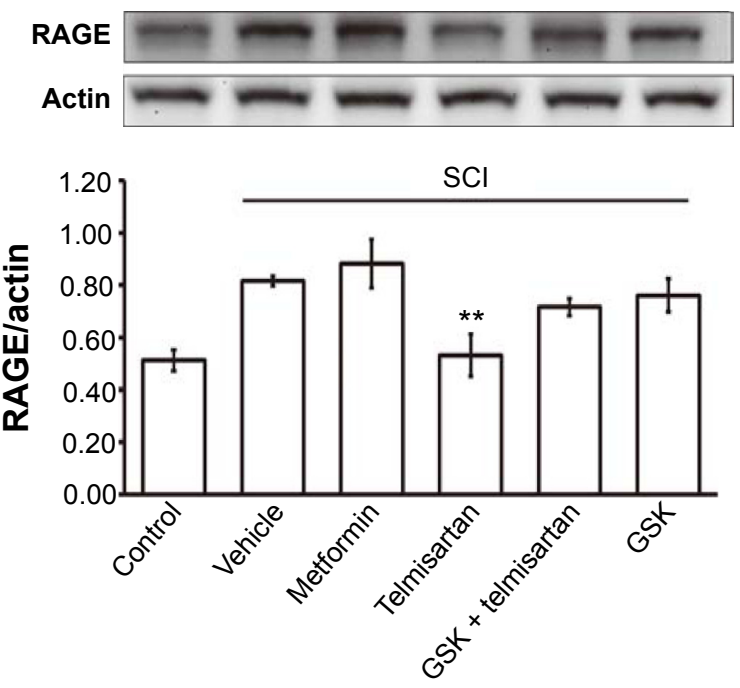

Figure 4 The expressions of HMGBI and RAGE in spinal cord from rats with SCl after treatment with metformin, telmisartan and/or GSK0660.

Notes: The rats with SCl were treated with metformin $(320 \mathrm{mg} / \mathrm{kg})$, telmisartan $(5 \mathrm{mg} / \mathrm{kg})$ and/or GSK0660 (0.I mg/kg) intravenously once daily for $28 \mathrm{days}$. Expressions of (A) HMGBI and (B) RAGE were identified using Western blotting analysis. Protein expression was normalized against that of $\beta$-actin used as a loading control. Data represent the mean \pm SEM of six animals. $* * P<0.01$ and $* * * P<0.00$ I compared with the vehicle-treated $\mathrm{SCl}$ rats.

Abbreviations: GSK, glycogen synthase kinase; HMGBI, high-mobility group box I protein; RAGE, receptor for advanced glycation endproducts; SCI, spinal cord injury; SEM, standard error of mean.

anti-inflammatory/antioxidant/anti-excitotoxic/pro-energetic profile in some neurological and inflammatory-related diseases. ${ }^{26}$ PPAR $\delta$ has also drawn much attention as a drug discovery target for regulating glucose and lipid metabolism ${ }^{27}$ because PPARs are widely distributed throughout the body and are mainly known for their effects on metabolism. Three isoforms of PPARs have been identified as PPAR $\alpha$, PPAR $\delta$ (also called PPAR $\beta$ ), and PPAR $\gamma .{ }^{28}$ PPAR $\alpha$ and PPAR $\gamma$ are involved in the metabolism of lipid and glucose. Basically, PPAR $\delta$ is the most abundant PPAR isoform in the body and many studies have demonstrated its role in antioxidative stress ${ }^{29}$ and neuroprotection. ${ }^{30}$ Moreover, decrease of PPAR $\delta$ delayed the recovery of motor behaviors in animals with SCI, which was related to a reduced ability to repair the injured tissue and to recover the neurological functions. ${ }^{31}$ As shown in Figures 1 and 2, rats with SCI showed poor motor behaviors than normal rats while the expression of PPAR $\delta$ in spinal cord was also decreased. Our results are consistent with the previous report ${ }^{9}$ showing the usefulness of this animal model.

Selective $\beta 1$-adrenoceptor antagonists have demonstrated a reduction in neurological injury in a rat model of spinal cord ischemia-reperfusion under its' effective dosage for treating hypertension. ${ }^{32}$ Additionally, calcium channel blocker has been identified to ameliorate the disease in a mouse model of multiple sclerosis. ${ }^{33}$

ARBs are also widely used for the treatment of hypertension in clinics. By blocking type-1 Ang II receptors, ARBs not only ameliorate hypertension but also inhibit the Ang II-stimulated induction of profibrotic cytokines and oxidative stress, thereby retarding the progression of chronic renal parenchymal injury. ${ }^{34}$ Some ARBs have also been shown to act as activators of PPAR and consequently induce anti-inflammatory effects by interfering with inflammatory signaling. ${ }^{35}$ Telmisartan, one of ARBs, has recently been attracting attention due to its putative PPAR $\delta$ action ${ }^{13}$ and ensuing anti-inflammatory effects. ${ }^{36,37}$ Previous report also indicated that telmisartan can activate the PPAR $\delta$ and increase the p-AMPK. ${ }^{13}$ However, the effect of ARBs in SCI is still unclear. Thus, we employed the same dose of telmisartan ${ }^{13}$ in our investigation in the present study.

In the central nervous system, PPAR $\delta$ is expressed mainly in oligodendrocytes and neurons. ${ }^{38}$ Activation of PPAR $\delta$ reduced the cellular and molecular changes occurring in SCI by targeting different downstream pathways, thereby modulating PPAR $\delta$ receptors. ${ }^{39}$ Also, this protective effect is significantly blocked by GSK0660. ${ }^{9}$ Comparable results were observed in the present study using rats with SCI, in which the PPAR $\delta$ expression and behavioral responses were both improved by telmisartan (Figures 1 and 2).

To evaluate the effects of motor function and pain responses in rats after SCI, several behavioral tests were applied, including the BBB locomotor scale, IPT, and limb hanging test. ${ }^{24,40,41}$ The motor functions in diabetic rats can be influenced after SCI by the pharmacological manipulation of PPAR $\delta$ activity, as described earlier. ${ }^{9}$ As shown in 
Figure 1, an activation of PPAR $\delta$ by telmisartan improved the motor dysfunction and increased pain sensitivity. Additionally, the blockade of PPAR $\delta$ using GSK0660 reversed these actions of telmisartan. Thus, PPAR $\delta$ is mediated in the telmisartan-induced recovery of damage in rats with SCI. It is also observed that PPAR $\delta$ is highly expressed in the central nervous system, ${ }^{42}$ with a great influence on neuronal cell functioning. ${ }^{43}$ Thus, we identified the changes of PPAR $\delta$ expression by telmisartan in rats with SCI. As shown in Figure 2, PPAR $\delta$ expression in spinal cords of these rats was markedly increased by telmisartan; this result is consistent with a previous reporton skeletal muscle. ${ }^{13}$ Our data shows that an activation of PPAR $\delta$ by telmisartan is helpfulin improving the injury from SCI in rats. However, more investigations are required in the future for understanding the molecular action mechanism(s) of PPAR $\delta$ in SCI.

Metformin has widely been used to treat type-2 diabetes patients. ${ }^{44-48}$ The key molecular mechanism of metformin is introduced to produce an activation of AMPK. ${ }^{36} \mathrm{~A}$ previous report has indicated that telmisartan can enhance the phosphorylation of AMPK through increased PPAR $\delta$ expression. ${ }^{13}$ It is similar to the present study using rats with SCI. However, the benefit of AMPK activation in the recovery of SCI is still obscure. In the present study, we used metformin as positive reference to test the role of p-AMPK in rats with SCI. The data showed that metformin failed to improve the motor behaviors (Figure 3) in SCI rats although it increased the expression of p-AMPK (Figure 2) similar to telmisartan. Metformin (the AMPK activator) treatment may not activate all the necessary factors and cofactors that mediate recovery in this compressive SCI model. Thus, mediation of AMPK seems not to be the major factor in the action of telmisartan for improvement of SCI in rats.

It has been indicated that ARBs inhibit the HMGB1/ RAGE axis ${ }^{49}$ to benefit patients with stroke. ${ }^{50}$ The extracellular release of HMGB1 appears to be common to the etiology and progression of multiple diseases leading to stroke, and may act in a multistage process in stroke. ${ }^{51}$ Inhibition of the HMGB1/ RAGE axis may prove to be a novel therapeutic strategy for treating stroke. ${ }^{52}$ This action was not related to the change of blood pressure by ARBs. ${ }^{52}$ Thus, it seems there is no correlation of SCI improvement and hypotensive effects in rats. Additionally, it has been demonstrated that activation of PPAR $\gamma$ inhibits the HMGB1-RAGE pathway. ${ }^{53,54}$ In the present study, we are the first to demonstrate that blockade of PPAR $\delta$ using GSK0660 can inhibit the telmisartan-induced changes of HMGB1 and RAGE expressions. However, the underlying mechanisms need more investigations in the future.

\section{Conclusion}

We found that telmisartan is effective in improving the motor behaviors in rats with compressive SCI through an increased expression of PPAR $\delta$ and a marked reduction in the expressions of HMGB1 and RAGE in spinal cords. In the current study, the therapeutic potential of telmisartan in SCI is addressed for the first time. However, more studies on the different models of SCI should be conducted in the future.

\section{Acknowledgments}

We thank Pei-Lin Chou and Yi-Zhi Chen for their assistance in our experiments.

\section{Disclosure}

The authors report no conflicts of interest in this work.

\section{References}

1. Ackery A, Tator C, Krassioukov A. A global perspective on spinal cord injury epidemiology. J Neurotrauma. 2004;21(10):1355-1370.

2. Hulsebosch CE. Recent advances in pathophysiology and treatment of spinal cord injury. Adv Physiol Educ. 2002;26(1-4):238-255.

3. Nath M, Wheeler JS Jr, Walter JS. Urologic aspects of traumatic central cord syndrome. J Am Paraplegia Soc. 1993;16(3):160-164.

4. Lamers C, Schubert-Zsilavecz M, Merk D. Therapeutic modulators of peroxisome proliferator-activated receptors (PPAR): a patent review (2008-present). Expert Opin Ther Pat. 2012;22(7):803-841.

5. Cuzzocrea S. Peroxisome proliferator-activated receptors and acute lung injury. Curr opin pharmacol. 2006;6(3):263-270.

6. Esposito E, Cuzzocrea S, Meli R. Peroxisome proliferator-activated receptors and shock state. Scientific World Journal. 2006;6: 1770-1782.

7. Di Paola R, Cuzzocrea S. Peroxisome proliferator-activated receptors ligands and ischemia-reperfusion injury. Naunyn Schmiedebergs Arch Pharmacol. 2007;375(3):157-175.

8. Ament Z, Masoodi M, Griffin JL. Applications of metabolomics for understanding the action of peroxisome proliferator-activated receptors (PPARs) in diabetes, obesity and cancer. Genome Med. 2012; 4(4):32.

9. Paterniti I, Esposito E, Mazzon E, et al. Evidence for the role of peroxisome proliferator-activated receptor-beta/delta in the development of spinal cord injury. J Pharmacol Exp Ther. 2010;333(2):465-477.

10. Kjeldsen SE, Julius S. Hypertension mega-trials with cardiovascular end points: effect of angiotensin-converting enzyme inhibitors and angiotensin receptor blockers. Am Heart J. 2004;148(5):747-754.

11. Mori Y, Itoh Y, Tajima N. Angiotensin II receptor blockers downsize adipocytes in spontaneously type 2 diabetic rats with visceral fat obesity. Am J Hypertens. 2007;20(4):431-436.

12. He H, Yang D, Ma L, et al. Telmisartan prevents weight gain and obesity through activation of peroxisome proliferator-activated receptor-deltadependent pathways. Hypertension. 2010;55(4):869-879.

13. Feng X, Luo Z, Ma L, et al. Angiotensin II receptor blocker telmisartan enhances running endurance of skeletal muscle through activation of the PPAR-delta/AMPK pathway. J Cell Mol Med. 2011;15(7): $1572-1581$.

14. Tsai CC, Lee KS, Chen SH, Chen LJ, Liu KF, Cheng JT. Decrease of PPARdelta in type-1-like diabetic rat for higher mortality after spinal cord injury. PPAR Res. 2014;2014:456386.

15. Chen SH, Yeh CH, Lin MY, et al. Premarin improves outcomes of spinal cord injury in male rats through stimulating both angiogenesis and neurogenesis. Crit Care Med. 2010;38(10):2043-2051. 
16. Rivlin AS, Tator CH. Effect of duration of acute spinal cord compression in a new acute cord injury model in the rat. Surg Neurol. 1978; 10(1):38-43.

17. Bikman BT, Zheng D, Kane DA, et al. Metformin improves insulin signaling in obese rats via reduced IKKbeta action in a fiber-type specific manner. J Obes. 2010;2010.

18. Shiota A, Shimabukuro M, Fukuda D, et al. Telmisartan ameliorates insulin sensitivity by activating the AMPK/SIRT1 pathway in skeletal muscle of obese db/db mice. Cardiovasc Diabetol. 2012;11:139.

19. Basso DM, Beattie MS, Bresnahan JC. A sensitive and reliable locomotor rating scale for open field testing in rats. J Neurotrauma. 1995; 12(1):1-21.

20. Rivlin AS, Tator $\mathrm{CH}$. Objective clinical assessment of motor function after experimental spinal cord injury in the rat. JNeurosurg. 1977;47(4): 577-581.

21. Pearse DD, Lo TP Jr, Cho KS, et al. Histopathological and behavioral characterization of a novel cervical spinal cord displacement contusion injury in the rat. J Neurotrauma. 2005;22(6):680-702.

22. Feldman P, Due MR, Ripsch MS, Khanna R, White FA. The persistent release of HMGB1 contributes to tactile hyperalgesia in a rodent model of neuropathic pain. J Neuroinflammation. 2012;9(1):180.

23. Metz GA, Merkler D, Dietz V, Schwab ME, Fouad K. Efficient testing of motor function in spinal cord injured rats. Brain Res. 2000; 883(2):165-177.

24. Pajoohesh-Ganji A, Byrnes KR, Fatemi G, Faden AI. A combined scoring method to assess behavioral recovery after mouse spinal cord injury. Neurosci Res. 2010;67(2):117-125.

25. Barez-Lopez S, Bosch-Garcia D, Gomez-Andres D, et al. Abnormal motor phenotype at adult stages in mice lacking type 2 deiodinase. PloS one. 2014;9(8):e103857.

26. Esposito E, Cuzzocrea S. Targeting the peroxisome proliferatoractivated receptors (PPARs) in spinal cord injury. Expert opinion on therapeutic targets. 2011;15(8):943-959.

27. Balakumar P, Rose M, Ganti SS, Krishan P, Singh M. PPAR dual agonists: are they opening Pandora's Box? Pharmacological Res. 2007;56(2): 91-98.

28. Qi C, Zhu Y, Reddy JK. Peroxisome proliferator-activated receptors, coactivators, and downstream targets. Cell Biochem Biophys. 2000; 32(Spring):187-204.

29. Lee H, Ham SA, Kim MY, et al. Activation of PPARdelta counteracts angiotensin II-induced ROS generation by inhibiting rac1 translocation in vascular smooth muscle cells. Free Radic Res. 2012;46(7): 912-919.

30. Schnegg CI, Robbins ME. Neuroprotective mechanisms of PPARdelta: modulation of oxidative stress and inflammatory processes. PPAR Res. 2011;2011:373560.

31. Wu QH, Chen WS, Chen QX, Wang JH, Zhang XM. Changes in the expression of platelet-derived growth factor in astrocytes in diabetic rats with spinal cord injury. Chin Med J. 2010;123(12):1577-1581.

32. Umehara S, Goyagi T, Nishikawa T, Tobe Y, Masaki Y. Esmolol and landiolol, selective beta1-adrenoreceptor antagonists, provide neuroprotection against spinal cord ischemia and reperfusion in rats. Anesth Analg. 2010;110(4):1133-1137.

33. Brand-Schieber E, Werner P. Calcium channel blockers ameliorate disease in a mouse model of multiple sclerosis. Exp Neurol. 2004; 189(1):5-9.

34. Nakao N, Sakai M, Fujimori A, Fukagawa M. Combination therapy with ACE inhibitors and angiotensin II receptor blockers in chronic renal disease: new therapeutic world beyond blood pressure reduction. Kidney Int. 2005;68(3):1375-1376.

35. Tan Y, Muise ES, Dai H, et al. Novel transcriptome profiling analyses demonstrate that selective peroxisome proliferator-activated receptor gamma (PPARgamma) modulators display attenuated and selective gene regulatory activity in comparison with PPARgamma full agonists. Mol pharmacol. 2012;82(1):68-79.
36. Matsui T, Yamagishi S, Ueda S, et al. Telmisartan, an angiotensin II type 1 receptor blocker, inhibits advanced glycation end-product (AGE)-induced monocyte chemoattractant protein-1 expression in mesangial cells through downregulation of receptor for AGEs via peroxisome proliferator-activated receptor-gamma activation. Jint Med Res. 2007;35(4):482-489.

37. Kimura H, Mikami D, Kamiyama K, et al. Telmisartan, a possible PPAR-delta agonist, reduces TNF-alpha-stimulated VEGF-C production by inhibiting the $\mathrm{p} 38 \mathrm{MAPK} / \mathrm{HSP} 27$ pathway in human proximal renal tubular cells. Biochem Biophys Res Commun. 2014;454(2):320-327.

38. Woods JW, Tanen M, Figueroa DJ, et al. Localization of PPARdelta in murine central nervous system: expression in oligodendrocytes and neurons. Brain Res. 2003;975(1-2):10-21.

39. Esposito E, Paterniti I, Meli R, Bramanti P, Cuzzocrea S. GW0742, a high-affinity PPAR-delta agonist, mediates protection in an organotypic model of spinal cord damage. Spine. 2012;37(2):E73-E78.

40. Sedy J, Urdzikova L, Jendelova P, Sykova E. Methods for behavioral testing of spinal cord injured rats. Neurosci Biobehav Rev. 2008;32(3): $550-580$.

41. Shinozaki M, Takahashi Y, Mukaino M, et al. Novel concept of motor functional analysis for spinal cord injury in adult mice. J Biomed Biotechnol. 2011;2011:157458.

42. Hall MG, Quignodon L, Desvergne B. Peroxisome proliferator-activated receptor beta/delta in the brain: facts and hypothesis. PPAR Res. 2008;2008:780452.

43. Iwashita A, Muramatsu Y, Yamazaki T, et al. Neuroprotective efficacy of the peroxisome proliferator-activated receptor delta-selective agonists in vitro and in vivo. J Pharmacol Exp Ther. 2007;320(3):1087-1096.

44. Smiechowski B, Azoulay L, Yin H, Pollak MN, Suissa S. The use of metformin and colorectal cancer incidence in patients with type II diabetes mellitus. Cancer Epidemiol Biomarkers Prev. 2013;22(10):1877-1883.

45. Bacha F, Pyle L, Nadeau K, et al. Determinants of glycemic control in youth with type 2 diabetes at randomization in the TODAY study. Pediatr Diabetes. 2012;13(5):376-383.

46. Han SJ, Choi SE, Kang Y, et al. Effect of sitagliptin plus metformin on beta-cell function, islet integrity and islet gene expression in Zucker diabetic fatty rats. Diabetes Res Clin Pract. 2011;92(2):213-222.

47. Hettihewa LM, Jayasinghe SS, Imendra KG, Weerarathna TP. Correlation between changes of blood pressure with insulin resistance in type 2 diabetes mellitus with 4 weeks of pioglitazone therapy. Int J Diabetes Dev Ctries. 2008;28(1):26-30.

48. Zhou G, Myers R, Li Y, et al. Role of AMP-activated protein kinase in mechanism of metformin action. J clin Investig. 2001;108(8): 1167-1174.

49. Hayakawa K, Miyamoto N, Seo JH, et al. High-mobility group box 1 from reactive astrocytes enhances the accumulation of endothelial progenitor cells in damaged white matter. J Neurochem. Epub 2012 Dec 10.

50. Wang L, Zhang X, Liu L, Yang R, Cui L, Li M. Atorvastatin protects rat brains against permanent focal ischemia and downregulates HMGB1, HMGB1 receptors (RAGE and TLR4), NF-kappaB expression. Neurosci Lett. 2010;471(3):152-156.

51. Rauvala H, Rouhiainen A. RAGE as a receptor of HMGB1 (Amphoterin): roles in health and disease. Curr Mol Med. 2007;7(8):725-734.

52. Kikuchi K, Tancharoen S, Ito $\mathrm{T}$, et al. Potential of the angiotensin receptor blockers (ARBs) telmisartan, irbesartan, and candesartan for inhibiting the HMGB1/RAGE axis in prevention and acute treatment of stroke. Int J Mol Sci. 2013;14(9):18899-18924.

53. Wang G, Liu L, Zhang Y, et al. Activation of PPARgamma attenuates LPS-induced acute lung injury by inhibition of HMGB1-RAGE levels. Eur J Pharmacol. 2014;726:27-32.

54. Wang G, Han D, Zhang Y, et al. A novel hypothesis: up-regulation of HO-1 by activation of PPARgamma inhibits HMGB1-RAGE signaling pathway and ameliorates the development of ALI/ARDS. J Thorac Dis. 2013;5(5):706-710. 
Drug Design, Development and Therapy

Dovepress

\section{Publish your work in this journal}

Drug Design, Development and Therapy is an international, peerreviewed open-access journal that spans the spectrum of drug design and development through to clinical applications. Clinical outcomes, patient safety, and programs for the development and effective, safe, and sustained use of medicines are a feature of the journal, which

has also been accepted for indexing on PubMed Central. The manuscript management system is completely online and includes a very quick and fair peer-review system, which is all easy to use. Visit http://www.dovepress.com/testimonials.php to read real quotes from published authors.

Submit your manuscript here: http://www.dovepress.com/drug-design-development-and-therapy-journal 